\title{
A Survey on Pneumatic Muscle Actuators Modeling
}

\author{
Eleni Kelasidi ${ }^{1}$, George Andrikopoulos ${ }^{1}$, George Nikolakopoulos ${ }^{2}$ and Stamatis Manesis ${ }^{1}$ \\ 1. Department of Electrical and Computer Engineering, University of Patras, Rio 26500, Achaia, Greece \\ 2. Department of Computer, Electrical and Space Engineering, Luleå University of Technology, Luleå 97187, Sweden
}

Received: October 18, 2011 / Accepted: January 05, 2012 / Published: September 30, 2012.

\begin{abstract}
The aim of this article is to provide a survey on the most popular modeling approaches for PMAs (pneumatic muscle actuators). PMAs are highly non-linear pneumatic actuators where their elongation is proportional to the interval pressure. During the last decade, there has been an increase in the industrial and scientific utilization of PMAs, due to their advantages such as high strength and small weight, while various types of PMAs with different technical characteristics have appeared in the literature. This article will: (a) analyse the PMA's operation from a mathematical modeling perspective; (b) present their merits and drawbacks of the most common PMAs; and (c) establish the fundamental basis for developing industrial applications and conducting research in this field.
\end{abstract}

Key words: Survey, modeling, PMA (pneumatic muscle actuator), pneumatic artificial muscle, fluidic muscle.

\section{Introduction}

Over the years, researchers have used various types of actuators on their industrial, robotic and automation applications. Hydraulic, electric, magnetic and pneumatic actuators are some of the commonly utilized types, with respect to the application's characteristics, function and limitations. During the last decade, there has been an increase in the use of pneumatic actuators in the industrial and medical areas, mainly due to their advantages such as low power to weight ratio, high strength and small weight [1].

Pneumatic muscle actuator [2], also known as the McKibben PAM (pneumatic artificial muscle) [3-6], fluidic muscle [7] or a biomimetic actuator [8], was first invented in 1950s by the physician, Joseph L. McKibben and was used as an orthotic appliance for polio patients [3]. PMAs are well suited for the implementation of positive load feedback, which is known to be used by animals. They present smooth, accurate and fast response and also produce a significant force when fully stretched. Moreover,

Corresponding author: Stamatis Manesis, professor, research fields: automatic control systems, industrial automation, mechatronics. E-mail: stam.manesis@ece.upatras.gr.
PMAs are lightweight, which is a particularly useful feature when working with applications that place restrictions on the weight of the equipment e.g., mobile robotic applications [9].

Until now PMAs were commonly used in areas like those of medical, industrial and entertainment robotics. Specifically, PMAs have been widely used in rehabilitation engineering as an actuator in orthotic exoskeleton appliances [10-12]. Artificial muscles have also been used in biomimetic robotics $[13,14]$ and for the development of artificial fine-motion limbs [13, 15]. PMAs can also be found in manufacturing, laboratory and aerospace applications [16].

Typical manufacturing of a PMA can be found as a long synthetic or natural rubber tube, wrapped inside man-made netting, such as Kevlar, at predetermined angle. Protective rubber coating surrounds the fibber wrapping and appropriate metal fittings are attached at each end. PMA converts pneumatic power to pulling force and has many advantages over conventional pneumatic cylinders such as high force to weight ratio, variable installation possibilities, no mechanical parts, lower compressed air consumption and low cost [17]. When compressed air is applied to the interior of the 
rubber tube, it contracts in length and expands radially. As the air exits the tube, the inner netting acts as a spring that restores the tube in its original form. This actuation reminds the operation of a single acting pneumatic cylinder with a spring return, while this reversible physical deformation during the contraction and expansion of the muscle results in linear motion. Recently PMAs have found significant medical applications [18-20] as PMA actuator resembles the function of a human muscle, and thus has been given the name pneumatic artificial muscle [4, 21]. It should be noted that the most significant advantage of utilizing PMA in control applications, is that for their position control, only one analog variable needs to be controlled, while for the same operation with a pneumatic cylinder, two analog variables need to be controlled (one for each chamber). As a result in the case of a pneumatic cylinder, it is more difficult to find an equilibrium between the two gauge pressures in the chambers, that it is for the case of PMAs.

As this type of actuators is becoming increasingly popular, many alternative types of PMAs have appeared in the scientific literature that differs in their mechanical construction and in the mathematical model describing their principal operation. Until now, except from the Festo AG Company's PMAs [4, 22-24], there has not been standardized and commercial versions of PMAs and in applications containing such actuators, is of paramount importance to: (1) choose PMA that fits the application, and (2) utilize the correct mathematical PMA model. The high non-linearities due to: (1) the existence of the pressurized air; (2) the elastic-viscous material; and (3) the geometric features of PMA, are the first problem that a control engineer will have to deal with in order to derive and utilize a proper PMA mathematical model.

PMAs can be addressed in the literature with different names, like fluidic muscle [25-27], air muscle [28, 29], pneumatic muscle actuator [29], fluid actuator [24], fluid-driven tension actuator [4], axially contractible actuator [30, 31], tension actuator [32, 33],
PPAM (pleated pneumatic artificial muscle) [34, 35], biomimetic actuator [34], BPA (braided pneumatic actuator) [36-40], paynter hyperboloid muscle [27, 41, 42], ROMAC (robotic muscle-like actuator) muscle $[34,42]$, and yarlott netted muscle [7, 42, 43]. Typical types of PAMs and the corresponding naming are depicted in Fig. 1.

The aim of this article is to provide an up-to-date literature review of the various manufacturing types of PMAs and their operation, while focusing on analysing the most popular and functional mathematical models of PMAs that can be found in the scientific literature. The survey, in this promising technology of PMAs, will also provide a wide set of the most important references in this field that could be further investigated by the interested readers.

This article is organized as follows. In Section 2, the basic PMA's principles of operation are presented. The most significant modeling approaches for PMAs are presented in Section 3. Finally, some conclusions are drawn in Section 4.

\section{Principles of Operation}

The basic principles of the PMA's operation can be categorized in two cases: (1) under a constant load and with varying gauge pressure, and (2) with a constant gauge pressure and a varying load. To illustrate this operation, a PMA of an arbitrary type is considered [34]. In the first case (Fig. 2), PMA is fixed at one end and has a constant mass load hanging from the other side. The pressure difference across the membrane, i.e., its gauge pressure, can be increased from an initial value of zero. At zero gauge pressure the volume enclosed by the membrane is minimal $V_{\min }$ and its length maximal $l_{\max }$. If the actuator is pressurized to some gauge pressure $P_{1}$, it will start to bulge and at the same time develop a pulling force that will lift the mass until the equilibrium point where the generated force will equal to the mass weight $M g$.

At this point the PMA's volume will have been increased to $V_{1}$ and its length contracted to $l_{1}$. 


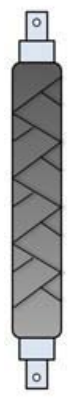

(a)

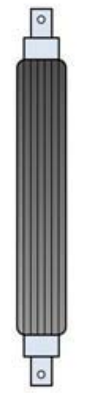

(b) (c)

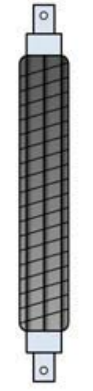

(d)

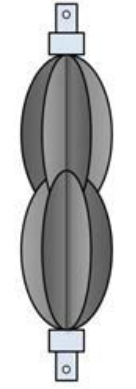

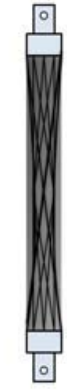

(e)
Fig. 1 Various types of PMAs: (a) McKibben muscle/braided muscle; (b) pleated muscle; (c) yarlott netted muscle; (d) ROMAC muscle; and (e) paynter hyperboloid muscle.

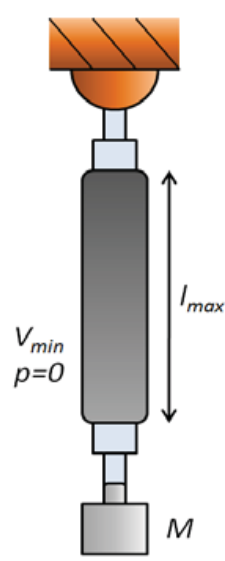

(a)

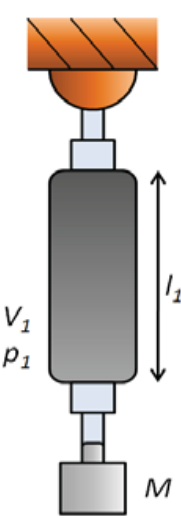

(b)

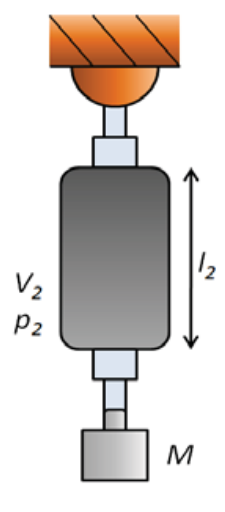

(c)
Fig. 2 PMA operation at constant load.

Increasing the pressure further to $P_{2}$ will continue this process, until the gauge pressure reaches the maximum allowable value $P_{\max }$. During this type of operation PMA: (1) will shorten its length by increasing its enclosed volume, and (2) will contract against a constant load if the pneumatic pressure is increased.

The second type of PMA's operation, which is the case of operation under constant gauge pressure, is depicted in Fig. 3. In this case, the gauge pressure is kept at a constant value $P$, while the load is decreasing, driving the PMA to inflate and decrease its initial length $l_{3}$. If the load is completely removed, the swelling goes to its full extent, at which point the volume will reach its maximum value $V_{\max }$, the length of its minimal value $l_{\min }$, and the pulling force will drop to zero. PMA will not be able to contract beyond

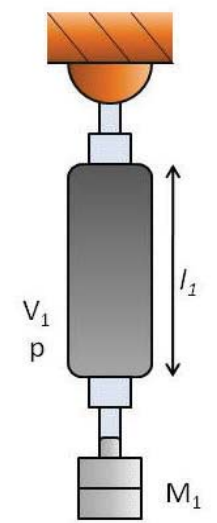

(a)

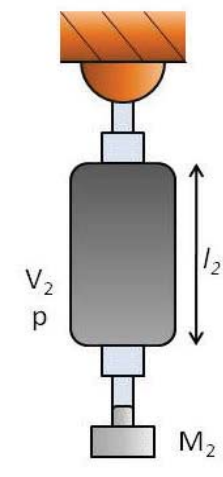

(b)

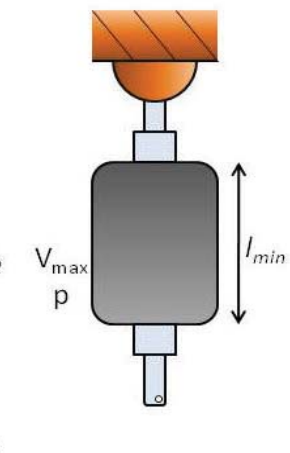

(c)
Fig. 3 PMA operation at constant pressure.

this point and it will operate as a bellows at shorter lengths, generating a pushing instead of pulling force. During this type of operation PMA: (1) will decrease its length at a constant pressure if its load is decreased, and (2) its contraction has an upper limit at which it develops no force and its enclosed volume is maximal.

For these two principal operations, it should be highlighted that for each pair of pressure and load, PMA has an equilibrium length. This characteristic is in a total contrast to the operation of a pneumatic cylinder where the developed actuation force only depends on the pressure and the piston surface area so that at a constant pressure, it will be constant regardless of the displacement [1].

\section{Modeling Approaches}

In recent years, there has been quite a lot of activity regarding the mathematical modeling of PMAs. The aim of such a model is to relate the pressure and length of the pneumatic actuator to the force it exerts along its entire axis. In the process of deriving a proper PMA model, variables such as pulling force, actuator's length, air pressure, diameter and material properties, play a major role in the PMA dynamical behavior and this is why the mathematical models aim to describe the relationships between these factors. Understanding those relationships is of paramount importance in every application that consists of PMAs and especially if the 
main goal is to control its overall function (mainly the length of actuation). Unfortunately, PMAs evidence strong non-linear force-length characteristics that make it more difficult to control them and obtain the demanded performance features [33, 44]. In the following subsections an analysis to the most common and valuable PMA's models will be presented.

\subsection{Geometrical Model of PMA}

The original method of modeling was based on the geometry of PMA, an approach that has not been very useful for predicting the dynamical characteristics of PMAs because their parameters are not easily measured during actuation. Thus, various different geometrical models were proposed to describe the behavior of PMA. Among these models, the Chou and Hannaford model [45] and the Tondu and Lopez model [46] have been widely used.

The Chou and Hannaford model is the simplest geometrical model for a static performance of a PMA. The proposed model is valid under the following assumptions: (1) the actuator is cylindrical in shape; (2) the threads in the sheath are inextensible and always in contact with the outside diameter of the latex bladder; (3) frictional forces between the tubing and the sheath and between the fibers of the sheath are negligible; and (4) latex tubing forces are negligible.

With this approach the PMA actuator can be modeled as a cylinder, depicted in Fig. 4, with a length $L$, thread length $b$, diameter $D$, and number of thread turns $n$. The angle $\theta$ is defined as the angle of the threads with the longitudinal axis [47].

When the PMA actuator inflates, $D$ and $L$ change, while $n$ and $b$ remain constant, while the expressions for the PMA's length and diameter can be formulated as:

$$
L=b \cos \theta, D=b \frac{\sin \theta}{n \pi}
$$

By combining Eq. (1) the thread length can be calculated as:

$$
b=\left(L^{2}+D^{2} n^{2} \pi^{2}\right)^{1 / 2}
$$

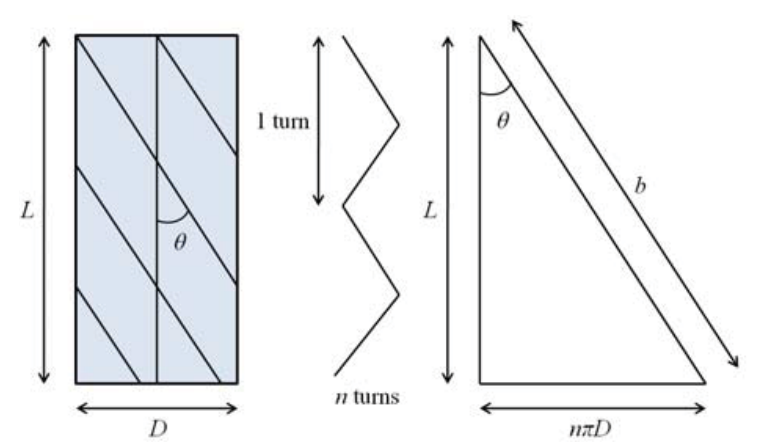

Fig. 4 Simplified geometrical model of PMA.

Eq. (2) is referred in the literature as the geometric relationship for PMA, while its volume is provided by:

$$
V=\frac{b^{3} \cos \theta \sin ^{2} \theta}{4 n^{2} \pi}
$$

Utilizing the energy conservation principle, PMA simple geometric force $F_{g}$ can be calculated as the gauge pressure multiplied by the change in volume with respect to length (this model can also be found in [47]):

$$
F_{g}=\frac{p b^{2}\left(3 \frac{L^{2}}{b^{2}}-1\right)}{4 n^{2} \pi}
$$

Another simple and widely-used geometrical model of PMA is that of Tondu and Lopez [46]. Based on this approach and by: (1) utilizing similar geometric description of the muscle [45]; (2) assuming inextensibility of the mesh material; and (3) angle changes during the alteration of PMA's length, the following expression of the contraction force $F$ generated by the muscle, as a function of the control pressure $P$ and the contraction ratio $\varepsilon$, based on the theorem of virtual work, is deduced $[46,48,49]$ :

$$
F(\epsilon, P)=\pi r_{0}^{2} \times p\left[\theta(1-\epsilon)^{2}-\beta\right]
$$

where:

$$
\epsilon=\frac{l_{0}-1}{l_{0}}, 0 \leq \epsilon \leq \epsilon_{\max }, \theta=\frac{3}{\tan ^{2} \theta_{0}} \beta=\frac{1}{\sin ^{2} \theta_{0}}
$$

In Eqs. (5) and (6), $r_{0}$ is the nominal inner radius, $l$ is the length of the muscle, $l_{0}$ is the initial nominal length, $P$ is the pressure and $\theta_{0}$ is the initial angle between the membrane fibres and the muscle axis, while this model can also be found in Ref. [21]. 
A disadvantage of the model is that its design is based on the hypothesis of a continuously cylindrical-shaped muscle, whereas it takes a conic shape at both ends when it contracts. Consequently, the more the muscle contracts, the more its active part decreases. This phenomenon results in the actual maximum contraction theoretically being smaller than that expected from Eq. (5) [50]. These models still have limitations in predicting the behavior of PMA in no-load conditions. However, there is a major phenomenon which Tondu and Lopez [46] considered to improve Eq. (5), which is the addition of an empirical correction factor $k$ to account for an end deformation of PMA:

$$
F(\epsilon, P)=\pi r_{0}^{2} \times P\left[\theta(1-k \epsilon)^{2}-\beta\right]
$$

where again: $0 \leq \epsilon \leq \epsilon_{\max }$ and $\epsilon_{\max }$ is provided from:

$$
\epsilon_{\max }=(1 / k)(1-\sqrt{\beta / \theta})
$$

Inserted in this way within the considered static model, the parameter $k$ does not modify the value of the maximum force given at zero contraction ratio. This is in concordance with the conducted experiment since PMA has a cylindrical shape only when its contraction ratio is zero. Furthermore, the parameter $k$ allows adapting the model maximum contraction ratio given by Eq. (8) to the experimental data. Thus, it tunes the "slope" of the considered static model.

In addition it has established two options for the selection of the parameter $k$ : (1) a constant value which may vary depending on the material that the muscle is made of, and (2) the parameter $k$ depends on the pressure in the muscle at any given time. It has been observed in Refs. $[45,46]$ that during operation there is a force/displacement hysteresis in the muscles caused by friction between the braid stands. Chou and Hannaford [45] produced a model including an experimentally obtained force offset which was added to calculate forces during muscle contraction and subtracted during extension. Tondu and Lopez [46] took this concept further by attempting to quantify the offset force by modeling the friction. Although the model produced was more accurate than that of Chou and Hannaford, it still relied on a degree of experimental data. In Ref. [51], Chou and Hannaford proposed that physical configuration and the behavior of PMA hinted the variable stiffness similar to spring-like characteristics.

In Ref. [52], more realistic muscle geometry has been utilized, in order to model the muscle's true form, by taking into consideration the irregular shape of the end sections during inflation. The proposed geometry includes a frustum cone that models each end section and a cylinder to model the muscle middle section. The middle-section geometry is governed by the deformation of the braid and its relationship is based on the form already shown in Fig. 4. With respect to the geometrical model depicted in Fig. 5, $L$ is the overall muscle length, $L_{L}$ is the horizontal length of the cone, $L_{Z}$ is the cone generator length, $\beta$ is the frustum-cone angle of the muscle ends, $L_{m}$ is the middle-section length, $D$ is the middle section diameter and $d$ is the end-fixture diameter.

All parameters are a function of the muscle-contracted length, except $L_{Z}$ that is determined experimentally based on the muscle end diameter and maximum contracted diameter. The relationships between the muscle diameter $D$ and the muscle middle-section length $L_{m}$ are again provided by Eq. (1), where $b, \theta$ and $n$ were previously described in Fig. 4. By utilizing Eq. (1), the braid angle is eliminated and the braid diameter is calculated by:

$$
D=\frac{\left(b^{2}-L_{m}^{2}\right)^{1 / 2}}{\pi n}
$$

During contraction, the cone base diameter expands beyond the end-fixture diameter. The cone horizontal length $L_{L}$ is:

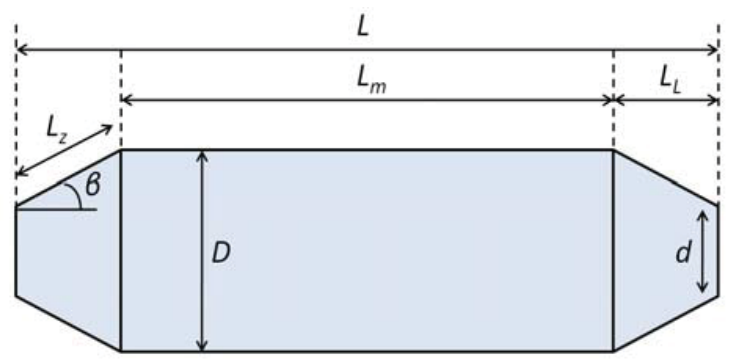

Fig. 5 Geometrical model of PMA. 


$$
L_{L}=\left(L_{z}^{2}-(D / 2-d / 2)^{2}\right)^{1 / 2}
$$

Finally, the total muscle length is:

$$
L=L_{m}+2 L_{L}
$$

If the muscle is assumed cylindrical, its length can be expressed by:

$$
L=\left(b^{2}-(\pi n D)^{2}\right)^{1 / 2}
$$

Another method of PMA modeling that can be found in literature is Biomimetic [34]. Due to the similarities between PMAs and biological muscle, this method attempts to apply models from biological muscle to PMAs [17].

\subsection{Phenomenological Biomimetic/Biomechanical Model}

The classic configuration of a PMA consists of a composite material that is made from a rubber mold with embedded structural fibers. When PMA under pressure extends over a circumferential direction, creating a force reduction in the longitudinal direction. The primary focus of the previous research was the development of geometrical model describing the behavior of PMAs. More recently, a phenomenological approach to model accurately describes the dynamics of PMAs using a model with three elements.

In the phenomenological model approach PMA is being considered as a parallel pattern that consists of a spring element, a damping element and a contractile element as it is presented in Fig. 6 [53]. The equation describing the motion for this model is:

$M \ddot{X}_{O-P M A}+B_{P M A} \dot{X}_{O-P M A}+K_{P M A} X_{O-P M A}=F_{c e}-L(13)$ where $X_{O-P M A}$ is the displacement of PMA, $M$ is the mass of moving parts, $B_{P M A}$ is the damping coefficient, $F_{c e}$ is the contractile force coefficient, $K_{P M A}$ is the spring coefficient of PMA and $L$ is the external load. Because of the low mass system, the element $M$ is negligible [19].

In Eq. (13), $f(t)=F_{c e}-L$ can be defined as the function that produces the input and causes the system to become operational and thus Eq. (13) can be cast as:

$$
B_{P M A} \frac{d X_{O-P M A}}{d t}+K_{P M A} X_{O-P M A}=f(t)
$$

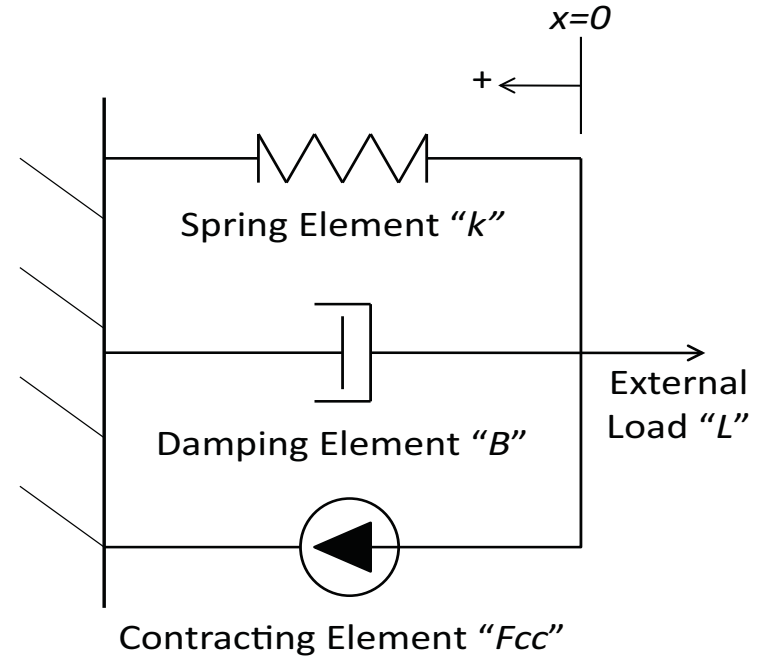

Fig. 6 Phenomenological model of PMA.

by dividing $K_{P M A}$, Eq. (14) becomes:

$$
\frac{B_{P M A}}{K_{P M A}} \frac{d X_{O-P M A}}{d t}+X_{O-P M A}=\frac{f(t)}{K_{P M A}}
$$

and finally by substituting $\tau=\left(B_{P M A} / K_{P M A}\right)$ and $f(t)=K_{P M A} X_{I-P M A}$, where $X_{I-P M A}$ is the desired displacement of PMA, Eq. (15) becomes:

$$
\tau \frac{d X_{O-P M A}}{d t}+X_{O-P M A}=X_{I-P M A}
$$

By taking the Laplace transform and setting the initial conditions to zero, the transfer function of the phenomenological model of PMA is derived as [53]:

$$
\frac{X_{O-P M A(S)}}{X_{I-P M A(S)}}=\frac{(1 / \tau)}{S+(1 / \tau)}
$$

\subsection{Mathematical Model of the Curved PMA}

Another type of a popular PMA configuration is the curved PMA. Although several studies have been performed for deriving classical PMA models that well describe the classical PMA configurations such as Mckibben muscle, braided muscle and so on, there is a gap in modeling curved PMAs as they operate with a different principle.

Schmidt [54] developed two approximate physics-based models: (1) the beam model; and (2) the membrane model for modeling the tube bending. These 
two models may represent opposite ends of the process governing the mobility of the curved PMA. The beam model assumes that the power/torque output of the curved PMA rotary actuator is determined exclusively by elastic deformation without change in the internal volume of the curved part of PMA, while the membrane model makes the opposite assumptions. The curved PMA does not stretch, although the cross sectional shape deforms, thereby reducing the internal volume.

The beam model treats PMA which is under pressure as a long, thin part, loaded in a single plane, and with a mechanical behavior governed by the elasticity and bending phenomena. The central idea of the above model is that the fabric can only support extensional stresses [50]. The relationship between the curvature of the beam, the applied moment $M$, the initial pressure $P$ and the radius of the beam $r$, can be expressed by the following equation:

$$
M=\operatorname{Pr}^{3} \frac{\frac{\pi}{2}[(\pi-\varphi)+\sin \varphi \cos \varphi]-v\left[(\pi-\varphi)^{2}-(\pi-\varphi) \sin \varphi \cos \varphi-(2 \sin \varphi)^{2}\right]}{\sin \varphi+(\pi-\varphi) \cos \varphi}
$$

where $\varphi$ is the curved angle and $v$ is the Poisson's ratio of PMA.

The membrane model uses a principle that relates the time to apply the bending moment, the force applied at the ends of curved PMA and the PMA's curved angle, when PMA is under pressure. The potential energy $E$ of the curved PMA, with a volume $V$, an initial pressure $P$, a corresponding force $F$, a bending moment $M$, a curved angle $\varphi$, and a linear displacement $d$ along with the axis of the curved PMA (presented in Fig. 7) is given by [50] :

$$
E=-P V-F d-M \varphi
$$

At the equilibrium point of the system the potential energy is minimized with:

$$
\frac{d E}{d \varphi}=-p \frac{d V}{d \varphi}-\frac{F d}{d \varphi}-M=0
$$

When $F=0$ and $d=0$, the Eq. (20) becomes:

$$
M=-p \frac{d V}{d \varphi}
$$

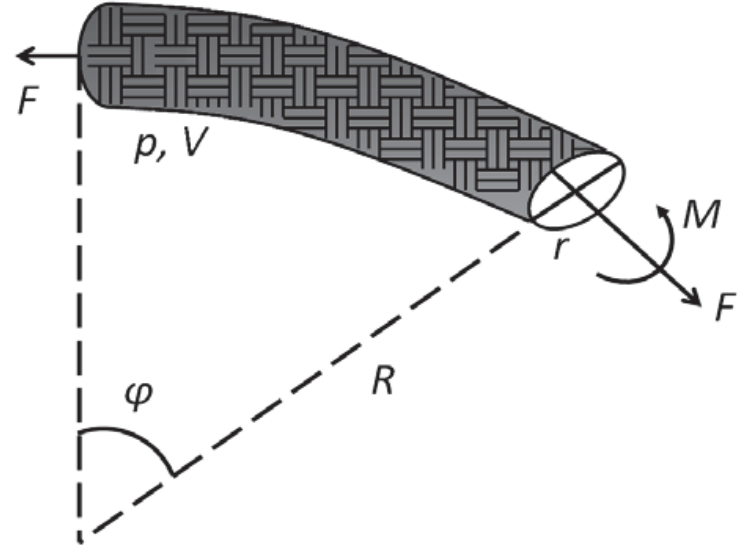

Fig. 7 Curved PMA according to beam and membrane model.

Eq. (21) shows that the relationship between equilibrium bending moment and curved angle is being determined by the relationship between the internal volume of the tube and the curved angle:

$$
M=\beta \times M_{B}+(1-\beta) \times M_{M}
$$

Eq. (22) presents the relationship between the previous two models by the introduction of a weight factor $\beta$. When the actuator behaves as a beam model then $\beta=1$ and when the behavior is close to the membrane model then $\beta=0 . M_{B}$ represents the moment generated as the beam model and $M_{M}$ represents the moment generated as the membrane model.

Based on experimental results in Ref. [50], it was found that the actuator produces increased torque outputs by correspondingly increasing the curved angle, the internal air pressure, the internal radius, and decreasing the curvature of PMA.

\subsection{Empirical Model of PMA}

PMAs of different lengths and diameters can also be modeled by simplified empirical models. In this approach, initially PMA is considered to have the real length at zero gauge pressure or at atmospheric pressure without any load to pull, and a nominal length $L_{0}$. When the air pressure $P$ inside PMA changes from low pressure $P_{1}$ to high pressure $P_{2}$, PMA contracts by moving towards a new equilibrium length 
(Fig. 8a). In this modeling approach the maximum PMA's length during contraction is depended on the air pressure and is called unstretched length and is being indicated as $L_{u}$ and it can be proven [55] that $L_{u}$ is changed according to the air pressure. As it is presented in Fig. 8b, PMA has the contractile tend to increase its length according to the power of attraction. If the instantaneous muscle length is $L$, the stretched length $L_{s}$, can be defined as the difference between the instantaneous length and the un-stretched length. It should be noted that PMA has a certain working length for a given pressure. For example, the stretched length is zero when the air pressure inside PMA is the atmospheric pressure and the stretched length increases when the air pressure also increases.

As cited in Refs. [55, 56], the functionalities of PMAs and of the mechanical springs, have fairly similar characteristics, when operated with or without pulling forces in working conditions. The natural mechanical operation of these two systems can be presented as is depicted in Fig. 9, where PMA and the mechanical spring exhibit similar behavior when a tensile force is being applied to them. Conventionally, the stiffness of the spring is fixed and depends on the material properties and geometry of the spring. On the other hand, the stiffness of PMA is variable and depends not only on the above properties but also on the air pressure inside PMA.

Based on experimental observations [56], the pulling force acting on PMA can be modeled in the same way as force acting on a mechanical spring. The parameter of stiffness in a PMA can also be indicated as $K$ and is a function of the operating gauge pressure $P$ and the stretched length $L_{s}$, defined as $L_{s}=L-L_{u}$, while the un-stretched length can be calculated as in Eq. (23) and by utilizing the least squares method to find the values of the constant parameters $h_{1}, h_{2}, h_{0}$ :

$$
L_{u}=h_{2} P^{2}+h_{1} P+h_{0}
$$

The elastic force generated by PMA is indicated as $F_{\text {elastic }}$ and its relationship to the previous parameters $\left(K, L_{s}\right)$ is presented in Eq. (24):

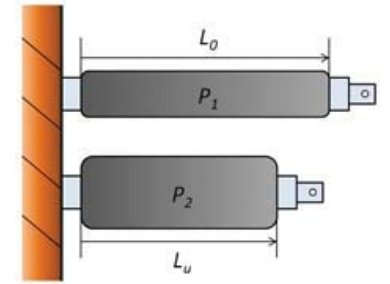

(a)

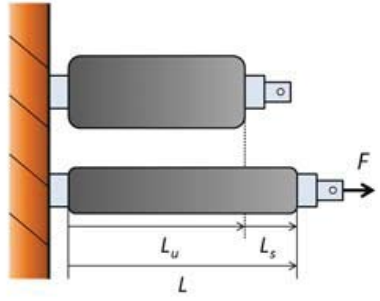

(b)
Fig. 8 Parameter of PMA: (a) initial and final length of PMA, and (b) length definition when PMA is extended by pulling force.

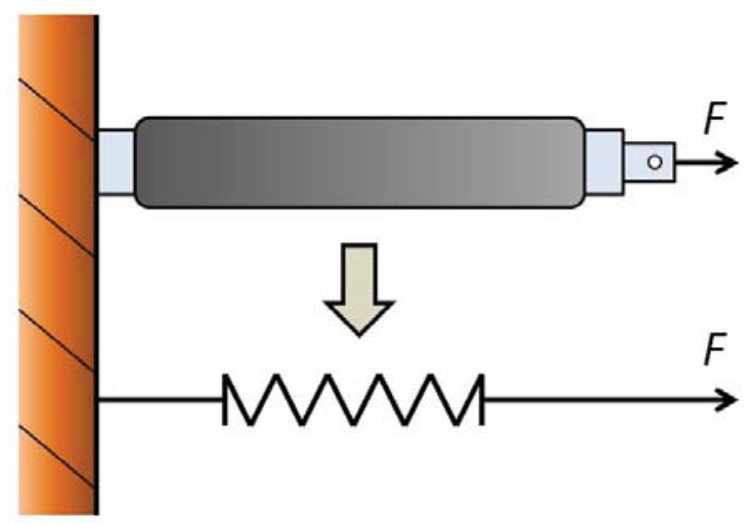

Fig. 9 Diagram of PMA and spring system.

$$
F_{\text {elastic }}=K\left(P, L_{s}\right) L_{s}
$$

In Eq. (24), the stiffness $K$, explicitly has been considered as a second order polynomial of $P$ and $L_{s}$, as it is presented in Eq. (25):

$$
K=c_{3} P^{2}+c_{2} P L_{s}+c_{1} L_{s}^{2}+c_{0}
$$

where the parameters $c_{1}, c_{2}, c_{3}, c_{0}$ are constants and can be found from experimental data via applying the method of least squares $[55,56]$.

Higher degree polynomials can be further applied by utilizing the same approach, with respect to the desired precision requirement. From observation results showed in Refs. [55, 56], the stiffness value is increasing while the stretched length also increases for a given air pressure. However, it is observed that for a given stretched length, the stiffness decreases when the air pressure inside PMA also increases. On the other hand, the stiffness parameter $K$ increases when the air pressure inside PMA is greater than a minimum pressure value. This unexpected non-linear behavior is 
produced as the effect of the complex PMA mechanical structure, the geometry, and elastic material itself.

\section{Conclusions}

In this article, a survey on the modeling approaches for PMAs has been presented. The most popular models of PMAs including: (1) the geometrical, (2) the phenomenological, (3) the curved, and (4) the empirical models have been presented and analysed towards their merits and disadvantages. A significant reference list has been provided as a basis for further studies and investigations in the most promising field of PMAs.

\section{References}

[1] F. Daerden, D. Lefeber, Pneumatic artificial muscles: Actuators for robotics and automation, European Journal of Mechanical and Environmental Engineering 47 (2002) 10-21.

[2] D. Caldwell, G. Medrano-Cerda, M. Goodwin, Braided pneumatic actuator control of a multi-jointed manipulator, in: Proceedings of the IEEE International Conference on Systems, Man and Cybernetics, Le Touquet, 1993, pp. 423-428.

[3] V. Nickel, J. Perry, A. Garrett, Development of useful function in the severely paralyzed hand, Journal of Bone and Joint Surgery 45A (5) (1963) 933-952.

[4] H. Schulte, The Characteristics of the McKibben Artificial Muscle, in: The Application of External Power in Prosthetics and Orthotics, National Academy of Science, NRC, Appendix H, 1961, pp. 94-115.

[5] G.K. Klute, J.M. Czerniecki, B. Hannaford, McKibben artificial muscles: Pneumatic actuators with biomechanical intelligence, in: IEEE/ASME International Conference on Advanced Intelligent Mechatronics, Atlanta, USA, 1999, pp. 221-226.

[6] G.K. Klute, B. Hannaford, Accounting for elastic energy storage in McKibben artificial muscle actuators, ASME Journal of Dynamic Systems, Measurement and Control 122 (2) (2000) 386-388.

[7] J. Yarlott, Fluid actuator, US Patent, 3645173 (1972).

[8] D. Caldwell, N. Tsagarakis, Biomimetic actuators in prosthetic and rehabilitation applications, Technology and Health Care 10 (2) (2002) 107-120.

[9] D. Caldwell, N. Tsagarakis, D. Badihi, G. Medrano-Cerda, Pneumatic muscle actuator technology: A lightweight power system for a humanoid robot, in: Proceedings of the IEEE International Conference on Robotics and
Automation, Leuven, 1998, pp. 3053-3058.

[10] T. Noritsugu, M. Takaiwa, D. Sasaki, Development of power assist wear using pneumatic rubber artificial muscles, in: Proc. of Asia Int. Symposium on Mechatronics, Tokyo, Japan, 2008.

[11] C. Vimieiro, B.G. Nascimento, D. Nagem, M. Pinotti, Development of a hip orthosis using pneumatic artificial muscles, in: Technology Meets Surgery International, Sao Paulo, Brazil, 2005.

[12] M. Knestel, E. Hofer, S. KleeBarillas, R. Rupp, The artificial muscle as an innovative actuator in rehabilitation robotics, The International Federation of Automatic Control, 2008.

[13] M.V. Damme, R.V. Ham, B. Vanderborght, F. Daerden, D. Lefeber, Design of a soft 2-DOF planar pneumatic manipulator, in: International Conference on Climbing and Walking Robots and the Support Technologies for Mobile Machines, 2005, pp. 559-566.

[14] D.A. Kingsley, R.D. Quinn, R.E. Ritzmann, A cockroach inspired robot with artificial muscles, in: International Symposium on Adaptive Motion of Animals and Machines (AMAM), Kyoto, Japan, 2003, pp. 559-566.

[15] P. Scarfe, E. Lindsay, Air muscles actuated low cost humanoid hand, Int. J. Advanced Robotic Systems 3 (2006) 139-146.

[16] N. Wereley, C. Kothera, E. Bubert, B. Woodes, M. Gentry, R. Vocke, Pneumatic artificial muscles for aerospace applications, in: 17th Conference on Structures, Structural Dynamics, and Materials, California, 2009.

[17] B. Hannaford, J.M. Winters, Actuator Properties and Movement Control: Biological and Technological Models, Springer Verlag, New York, 1990, pp. 101-120.

[18] G. Klute, J. Czerniecki, B. Hannaford, Muscle-like pneumatic actuators for below-knee prostheses, in: International Conference on New Actuators, 2000, pp. 289-292.

[19] J. Serres, D. Reynolds, C. Phillips, M. Gerschutz, D. Repperger, Characterisation of a phenomenological model for commercial pneumatic muscle actuators, Computer Methods in Biomechanics and Biomedical Engineering 12 (4) (2009) 423-430.

[20] T. Kerscher, J. Albiez, J. Zollner, R. Dillmann, Evaluation of the dynamic model of fluidic muscles using quick-release, in: The 1st IEEE/RAS-EMBS International Conference on Biomedical Robotics and Biomechatronics, Piza, Italy, 2006.

[21] F. Daerden, B. Verrelst, D. Lefeber, P. Kool, Controlling motion and compliance with folded pneumatic artificial muscles, in: Proceedings of the Second International Conference on Climbing and Walking Robots, Portsmouth, 1999, pp. 667-677.

[22] B. Tondu, V. Boitier, P. Lopez, Natural compliance of 
robot-arms based on McKibben artificial muscle actuators, in: Proceedings of the European Robotics and Intelligent Systems Conference, Malaga, 1994, pp. 783-797.

[23] B. Tondu, V. Boitier, P. Lopez, Theory of an artificial pneumatic muscle and application to the modelling of McKibben artificial muscle, French Academy of Sciences: Comptes Rendus, 1995, pp. 105-114.

[24] H. Baldwin, Realizable models of muscle function, in: Proceedings of the First Rock Biomechanics Symposium, New York, 1969, pp. 139-148.

[25] M. Kukolj, Axially contractible actuator, US Patent, 4733603 (1988).

[26] G. Immega, Tension actuator load suspension system, US Patent, 4826206 (1989).

[27] H. Paynter, Hyperboloid of revolution fluid-driven tension actuators and methods of making, US Patent, 4721030 (1988).

[28] J. Marcincin, A. Palko, Negative pressure artificial muscle-An unconventional drive of robotic and handling systems, Ph.D. Thesis, Transactions of the University of Kosice, Riecansky Science Publishing Co, Slovak Republic, 1993, pp. 350-354.

[29] T. McMahon, Muscles, Reflexes, and Locomotion, Princeton University Press, USA, 1984.

[30] M. Gavrilovic, M. Maric, Positional servo-mechanism activated by artificial muscles, Medical and Biological Engineering 7 (1969) 77-82.

[31] K. Inoue, Rubbertuators and applications for robotics, in: Proceedings of the 4th International Symposium on Robotics Research, New York, 1987, pp. 57-63.

[32] B. Hannaford, J. Winters, C. Chou, P. Marbot, The anthroform biorobotic arm: a system for the study of spinal circuits, Annals of Biomedical Engineering 23 (1995) 399-408.

[33] D. Caldwell, G. Medrano-Cerda, M. Goodwin, Control of pneumatic muscle actuators, IEEE Control Systems Magazine 15 (1) (1995) 40-48.

[34] F. Daerden, D. Lefeber, The concept and design of pleated pneymatic artificial muscles, International Journal of Fluid Power 2 (3) (2001) 41-50.

[35] B. Verrelst, F. Daerden, D. Lefeber, R.V. Ham, T. Fabri, Introducing pleated pneumatic artificial muscles for the actuation of legged robots: A one-dimensional set-up, in: Proceedings of the 3rd International Conference on Climbing and Walking Robots, Madrid, 2000, pp. 583-590.

[36] D. Caldwell, A. Razak, M. Goodwin, Braided pneumatic muscle actuators, in: Proceedings of the IFAC Conference on Intelligent Autonomous Vehicles, Southampton, 1993, pp. 507-512.

[37] J. Winters, Braided artificial muscles: Mechanical properties and future uses in prosthetics/orthotics, in:
Proceedings of the RESNA 13th Annual Conference, Washington DC, 1995, pp. 173-174.

[38] S. Davis, N. Tsagarakis, J. Canderle, D.G. Caldwell, Enhanced modeling and performance in braided pneumatic muscle actuators, International Journal of Robotics Research 22 (3) (2003) 213-227.

[39] D. Kingsley, R.D. Quinn, Fatigue life and frequency response of braided pneumatic actuators, in: IEEE Robotics and Automation Conference, Washington, 2002, pp. 2830-2835.

[40] R. Colbrunn, G. Nelson, R. Quinn, Modeling of braided pneumatic actuators for robotic control, in: Proceedings of 2001 IEEE/RSJ International Conference on Intelligent Robots and Systems, Maui, Hawaii, 2001, pp. 1964-1970.

[41] F. Daerden, Conception and realization of pleated pneumatic artificial muscles and their use as compliant actuation elements, Ph.D. Thesis, Vrije Universiteit Brussel, Belgium, 1999.

[42] D.V. Casi, Development of the production process of PPAM, Ph.D. Thesis, Universidad Publica de Navarra, 2009.

[43] H.M. Paynter, Low-cost pneumatic arthrobots powered by tug-and-twist polymer actuators, in: Japan/USA Symposium on Flexible Automation, 1996, pp. 107-110.

[44] T. Hesselroth, K. Sarkar, P.V. Smaght, K. Schulten, Neural network control of a pneumatic robot arm, IEEE Transactions on Systems, Man and Cybernetics 24 (1) (1994) 28-38.

[45] C. Chou, B. Hannaford, Measurement and modeling of McKibben pneumatic artificial muscles, IEEE Transactions on Robotics and Automation 12 (1) (1996) 90-102.

[46] B. Tondu, P. Lopez, Modeling and control of McKibben artificial muscle robot actuators, IEEE Control Systems Magazine 20 (2) (2000) 15-38.

[47] F. Daerden, D. Lefeber, P. Kool, Using free radial expansion pneumatic artificial muscles to control a 1DOF robot arm, in: Proceedings of the 1st International Symposium on Climbing and Walking Robots, Brussel, 1998, pp. 209-214.

[48] A. Pujana-Arrese, A. Mendizabal, J. Arenas, Modelling in Modelica and position control of a 1-DoF set-up powered by pneumatic muscles, Mechatronics 20 (5) (2010) 535-552.

[49] A. Pujana-Arrese, J. Arenas, I. Retolaza, A. Martinez-Esnaola, J. Landaluze, Modelling in Modelica of a pneumatic muscle: Application to model an experimental set-up, in: 21st European Conference on Modelling and Simulation, Prague, Czech Republic, 2007.

[50] J. Zhang, C. Yang, Y. Chen, Y. Zhang, Y. Dong, Modeling and control of a curved pneumatic muscle actuator for wearable elbow exoskeleton, Mechatronics 18 (8) (2008) 448-457. 
[51] C. Chou, B. Hannaford, Static and dynamic characteristics of McKibben pneumatic artificial muscles, in: Proceedings of IEEE International Conference on Robotics and Automation, San Diego, California, 1994, pp. 281-286.

[52] M. Doumit, A. Fahim, M. Munro, Analytical modeling and experimental validation of the braided pneumatic muscle, IEEE Transactions on Robotics 25 (6) (2009) 1282-1291.

[53] J. Serres, D. Reynolds, C. Phillips, D. Rogers, D. Repperger, Characterisation of a pneumatic muscle test station with two dynamic plants in cascade, Computer Methods in Biomechanics and Biomedical Engineering 13
(1) (2009) 11-18.

[54] P. Schmidt, An investigation of space suit mobility with applications to EVA operations, Ph.D. Dissertation, Massachusetts Institute of Technology, 2001.

[55] K. Wickramatunge, T. Leephakpreeda, Empirical modeling of pneumatic artificial muscle, in: Proceedings of International Multi Conference of Engineers and Computer Scientists, Hong Kong, China, 2009, pp. 1726-1730.

[56] K. Wickramatunge, T. Leephakpreeda, Study on mechanical behaviors of pneumatic artificial muscle, International Journal of Engineering Science 48 (2) (2010) 188-198. 